\title{
Dissipation through spin Coulomb drag in electronic spin transport and optical excitations
}

\author{
I. D'Amico ${ }^{1, *}$ and C. A. Ullrich ${ }^{2}$ \\ ${ }^{1}$ Department of Physics, University of York, York YO10 5DD, United Kingdom \\ ${ }^{2}$ Department of Physics and Astronomy, University of Missouri-Columbia, Columbia, Missouri 65211, USA
}

(Received 3 March 2006; revised manuscript received 1 August 2006; published 8 September 2006)

\begin{abstract}
Spin Coulomb drag (SCD) constitutes an intrinsic source of dissipation for spin currents in metals and semiconductors. We discuss the power loss due to SCD in potential spintronics devices and analyze in detail the associated damping of collective spin-density excitations. It is found that SCD contributes substantially to the linewidth of intersubband spin plasmons in semiconductor quantum wells, which suggests the possibility of a purely optical quantitative measurement of the SCD effect in a parabolic well through inelastic light scattering.
\end{abstract}

\section{DOI: 10.1103/PhysRevB.74.121303}

Spintronics applications are receiving increasing attention in the hope of revolutionizing traditional technology by a powerful exploitation of the spin-as well as the chargedegrees of freedom. An intense research effort is under way to improve our understanding of spin dynamics, especially related to nanocircuits and their components, such as quantum wells and wires. In this context the theory of spin Coulomb drag (SCD) was recently developed. ${ }^{1-5}$ This theory analyzes the role of Coulomb interactions between different spin populations in spin-polarized transport. Coulomb interactions transfer momentum between different spin populations, so that the total momentum of each spin population is not preserved. This provides an intrinsic source of friction for spin currents, a measure of which is given by the spin-transresistivity. ${ }^{1} \mathrm{SCD}$ is generally small in metals, due to a typical Fermi temperature of the order of $10^{5} \mathrm{~K}$, but can become substantial in semiconductors, where the spin transresistivity can be larger than the Drude resistivity. ${ }^{3,5}$ As the quest for defect-free materials with longer and longer spindecoherence times is continuing, spurred by practical requirements in spintronics as well as in quantum computation devices, the SCD is bound to become one of the most serious issues in spin-polarized transport, since, due to its intrinsic nature, it cannot be avoided even in the purest material. In fact, the recent experimental observation of SCD by Weber et al. ${ }^{6}$ shows that the effect dominates spin-diffusion currents over a broad range of parameters, in agreement with theoretical predictions. ${ }^{2,3,5}$

In this paper we discuss a critical issue for potential spintronics devices, namely, the power loss in spin transport and dynamics due to SCD. We shall analyze in detail its effect on optical spin excitations, and propose an experiment to measure the intrinsic SCD linewidth enhancement of spin plasmons in parabolic semiconductor quantum wells. While up to now SCD has been considered only in relation to spin transport, the proposed experiment would provide an alternative way of measuring this subtle effect, and thus establish unequivocally the influence of SCD on optical excitations.

Let us consider a system composed of spin-up and spindown electron populations, such as for example the electrons in the conduction band of a doped semiconductor structure. We are assuming spin-flip times long enough so that spin populations are well defined on the relevant time scales. This assumption-at the very core of spintronics-has been proved reasonable, with experimentally measured spin-
ACS number(s): 73.20.Mf, 73.21.-b, 73.40.-c, 73.50.-h

decoherence times of the order of microseconds. ${ }^{7}$ Previous papers on SCD have mainly analyzed the dependence of the spin transresistivity over temperature ${ }^{2-5}$ this paper will focus on its frequency dependence, ${ }^{1}$ which is important for both ac spintronics applications and spin-resolved optical experiments.

In the linear response regime and for weak Coulomb coupling one can write a phenomenological equation of motion for the spin- $\sigma$ population. ${ }^{1}$ The SCD force is defined as the Coulomb force (per unit volume) exerted by spin $\bar{\sigma}(=-\sigma)$ electrons, moving with center-of-mass velocity $\mathbf{v}_{\bar{\sigma}}$, on spin- $\sigma$ electrons, moving with center-of-mass velocity $\mathbf{v}_{\sigma}$ :

$$
\mathbf{F}_{\sigma \bar{\sigma}}(\omega)=-\gamma(\omega) m \frac{n_{\sigma} n_{\bar{\sigma}}}{n}\left(\mathbf{v}_{\sigma}-\mathbf{v}_{\bar{\sigma}}\right)
$$

where the number density $n_{\sigma}$ of $\sigma$-spin electrons of effective mass $m$ and the total density $n=n_{\uparrow}+n_{\downarrow}$ are those of a homogeneous reference system. The drag coefficient $\gamma$ appearing in Eq. (1) is directly proportional to the real part of the spin transresistivity $\rho_{\uparrow \downarrow}: 1$

$$
\gamma(\omega, T)=-\frac{n e^{2}}{m} \operatorname{Re} \rho_{\uparrow \downarrow}\left(\omega, T ; n_{\uparrow}, n_{\downarrow}\right),
$$

where $T$ is the electronic temperature. $\operatorname{Re} \rho_{\uparrow \downarrow}$ has a negative value and $\rho_{\uparrow \downarrow}$ can be defined through $\left.\mathbf{E}_{\uparrow}\right|_{\mathbf{j}_{\uparrow}=\mathbf{0}}=-e \mathbf{j}_{\downarrow} \rho_{\uparrow \downarrow}$, with $\mathbf{j}_{\sigma}$ the number current density of the $\sigma$-spin population, $\mathbf{E}_{\uparrow}$ the effective electric field which couples to the $\uparrow$-spin population and includes the gradient of the local chemical potential, and $e$ the absolute value of the electronic charge.

As noted above, SCD provides an intrinsic decay mechanism for spin-polarized currents, and is thus a source for power loss in a spintronics circuit or device. From the general definition of power and using Eq. (1), the SCD power loss density per unit time for the $\sigma$-spin population is given by

$$
\begin{aligned}
P_{\sigma}\left(\omega, n_{\uparrow}, n_{\downarrow}\right) & =\mathbf{F}_{\sigma \bar{\sigma}} \cdot \mathbf{v}_{\sigma} \\
& =e^{2}\left(\frac{n_{\bar{\sigma}}}{n_{\sigma}}\left|\mathbf{j}_{\sigma}\right|^{2}-\mathbf{j}_{\bar{\sigma}} \cdot \mathbf{j}_{\sigma}\right) \times \operatorname{Re} \rho_{\uparrow \downarrow}\left(\omega, T ; n_{\uparrow}, n_{\downarrow}\right) .
\end{aligned}
$$

Notice that $P_{\sigma}$ can change sign depending on the relative strength and direction of the spin-resolved current densities, a positive sign implying that the $\sigma$-spin population is being dragged along by the faster $\bar{\sigma}$-spin population. In particular, 


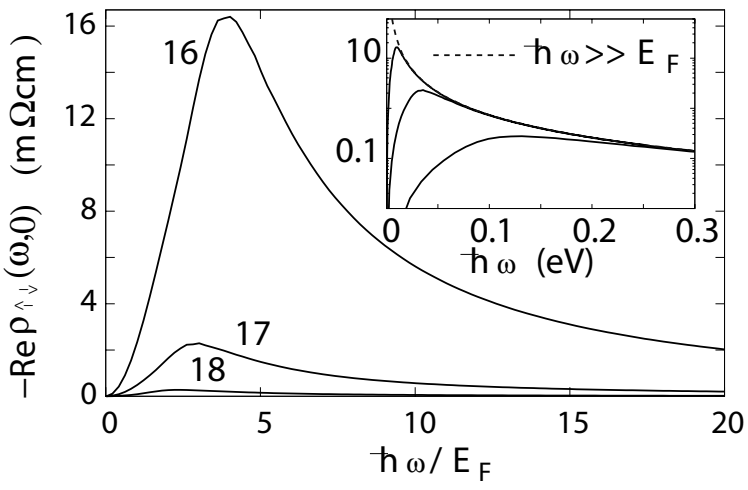

FIG. 1. Spin transresistivity $\left|\operatorname{Re} \rho_{\uparrow \downarrow}\right|$ vs rescaled frequency $\hbar \omega / E_{F}$ for $n=10^{x} \mathrm{~cm}^{-3}, x=16,17,18$ as indicated and GaAs parameters $\left(m=0.067 m_{e}, \epsilon=12\right)$. Inset: $\left|\operatorname{Re} \rho_{\uparrow \downarrow}\right|$ in $\mathrm{m} \Omega \mathrm{cm}$ vs $\hbar \omega$ in $\mathrm{eV}$ for the same densities. Dashed line: high-frequency limit Eq. (6).

for a system with spin populations drifting at the same average velocity, $P_{\sigma}(\omega)=0$. In a system with slowly varying density, we can use Eq. (4) to express the local power loss density in a volume element centered around position $\mathbf{r}$. The total power loss per unit time in the system can then be calculated as

$$
\bar{P}_{\sigma}(\omega)=\int_{V} d^{3} r\left[P_{\sigma}\left(\mathbf{r} ; \omega, n_{\uparrow}(\mathbf{r}), n_{\downarrow}(\mathbf{r})\right)\right] .
$$

Figure 1 shows the transresistivity $\operatorname{Re} \rho_{\uparrow \downarrow}\left(\omega ; n_{\uparrow}, n_{\downarrow}\right)$ as a function of frequency, calculated for GaAs at $T=0$, using a generalized random phase approximation. ${ }^{1}$ We see that $\operatorname{Re} \rho_{\uparrow \downarrow}$ has a maximum when $E_{F \sigma}\left(n_{\sigma}(z)\right)$ is of order $\hbar \omega\left(E_{F \sigma}\right.$ is the $\sigma$-spin Fermi energy). This maximum roughly scales $\operatorname{as}^{3}\left(h a^{*} / e^{2}\right) / n^{s} \approx 140 \mu \Omega \mathrm{cm} \times \epsilon m_{e} /\left(m n^{s}\right)$ with $s \lesssim 1$ : It is then reasonable to expect a sizable damping effect due to $\mathrm{SCD}$. We notice also that for very low densities, i.e., $E_{F}$ $\ll \hbar \omega$,

$$
\operatorname{Re} \rho_{\uparrow \downarrow}\left(\omega, T=0 ; n_{\uparrow}, n_{\downarrow}\right) \sim-\frac{\hbar a^{*}}{e^{2}}\left(\frac{2 \mathrm{Ry}^{*}}{\hbar \omega}\right)^{3 / 2} \frac{4 \pi}{3},
$$

independent of the carrier density (see Fig. 1 inset). ${ }^{15}$

To estimate the SCD dissipation, let $P_{\sigma}(\omega) \sim e^{2} j_{\sigma}^{2} \operatorname{Re} \rho_{\uparrow \downarrow}$ [see Eq. (4)]. For a GaAs ac spintronic device operating at terahertz frequencies around the maximum of $\operatorname{Re} \rho_{\uparrow \downarrow}$, with $j_{\sigma}=1 \mathrm{~A} / \mathrm{cm}^{2}$ and $n=10^{16} \mathrm{~cm}^{-3}\left(10^{18} \mathrm{~cm}^{-3}\right)$, we obtain $P_{\sigma}$ $\sim 16 \mathrm{~mW} / \mathrm{cm}^{3} \quad\left(0.28 \mathrm{~mW} / \mathrm{cm}^{3}\right)$. One finds $P_{\sigma} / P_{D}=25 \%$ (44\%), where $P_{D}(\omega) \sim e^{2} j_{\sigma}^{2} \rho_{D}$, and $\rho_{D}$ is the Drude resistivity associated with a mobility $10^{4} \mathrm{~cm}^{2} / \mathrm{V} \mathrm{s}$. This simple analysis shows that the dissipation from SCD and from impurities can be comparable. We expect an even higher SCD power loss in devices based on low-dimensional structures. ${ }^{5}$

Due to problems with electrical injection ${ }^{8}$ and the necessity of driving spin dynamics on subpicosecond time scales, ${ }^{9}$ much attention has been focused on optical spin injection ${ }^{7}$ and optically controlled spin dynamics; ${ }^{10}$ in the following, we will explore how the SCD affects the lifetime and dynamics of spin-dependent optical excitations.

The excitation spectrum of a system can be calculated in principle exactly with time-dependent density-functional theory (TDDFT). ${ }^{11}$ In TDDFT, an interacting time-dependent many-body system is described through a noninteracting Kohn-Sham system, characterized by an exchangecorrelation (XC) vector potential. The latter is a functional of the current, ${ }^{12}$ and needs to be approximated in practice.

Microscopically, the dissipation of spin currents can be viewed as loss of coherence due to decay into multiple particle-hole excitations of the underlying electronic manybody system. ${ }^{13}$ Describing these effects in TDDFT within a local approximation for the $\mathrm{XC}$ vector potential, one is naturally led to the language of hydrodynamics ${ }^{14}$ : dissipation arises from viscoelastic stresses in the electron liquid, which are proportional to the velocity gradients. This formal framework is dictated by global conservation laws and symmetries; however, the viscosity coefficients that are required as input come from detailed microscopic calculations. ${ }^{14}$ The corresponding XC potential for spin-dependent systems ${ }^{15}$ has an additional contribution accounting for the SCD.

Our derivation of the excitation energies for a spindependent system closely follows the spin-independent case. ${ }^{16}$ The starting point is the TDDFT current response equation

$$
\mathbf{j}_{\sigma}(\mathbf{r}, \omega)=\frac{e}{c} \int d^{3} r^{\prime} \underline{\underline{\chi}}_{\sigma}\left(\mathbf{r}, \mathbf{r}^{\prime}, \omega\right) \mathbf{a}_{\sigma}\left(\mathbf{r}^{\prime}, \omega\right) .
$$

Here, $\bar{\chi}_{\sigma}\left(\mathbf{r}, \mathbf{r}^{\prime}, \omega\right)$ is the Kohn-Sham current-current response tensor, which is diagonal in the spin channel. The effective vector potential is defined as $\mathbf{a}_{\sigma}=\mathbf{a}_{\sigma}^{\text {ext }}+\mathbf{a}_{\sigma}^{\mathrm{H}}+\mathbf{a}_{\sigma}^{\mathrm{XC}}$, where $\mathbf{a}_{\sigma}^{\text {ext }}$ is an external perturbation, and the Hartree and $\mathrm{XC}$ vector potentials are given by

$$
\begin{aligned}
& \frac{e}{c} a_{\nu \sigma}^{\mathrm{H}}(\mathbf{r}, \omega)=\frac{\nabla_{\nu}}{(i \omega)^{2}} \int d^{3} r^{\prime} \frac{\nabla^{\prime} \cdot \mathbf{j}\left(\mathbf{r}^{\prime}, \omega\right)}{\left|\mathbf{r}-\mathbf{r}^{\prime}\right|} \\
& \frac{e}{c} \mathbf{a}_{\nu \sigma}^{\mathrm{XC}}(\mathbf{r}, \omega)= \sum_{\sigma^{\prime}} \frac{\nabla_{\nu}}{(i \omega)^{2}} \int d^{3} r^{\prime} \nabla^{\prime} \cdot \mathbf{j}_{\sigma^{\prime}}\left(\mathbf{r}^{\prime}, \omega\right) f_{\mathrm{XC}, \sigma \sigma^{\prime}}^{\mathrm{ALDA}}\left(\mathbf{r}, \mathbf{r}^{\prime}\right) \\
&-\frac{1}{i \omega n_{\sigma}(\mathbf{r})} \sum_{\kappa \sigma^{\prime}} \nabla_{\kappa} \sigma_{\nu \kappa, \sigma \sigma^{\prime}}^{\mathrm{XC}}(\mathbf{r}, \omega) \\
&-\frac{e^{2}}{\omega} n_{\sigma}(\mathbf{r}) n_{\bar{\sigma}}(\mathbf{r}) \rho_{\uparrow \downarrow}\left(\omega ; n_{\sigma}(\mathbf{r}) n_{\bar{\sigma}}(\mathbf{r})\right) \\
& \times \sum_{\sigma^{\prime}} \frac{\sigma \sigma^{\prime}}{n_{\sigma}(\mathbf{r}) n_{\sigma^{\prime}}(\mathbf{r})} j_{\nu \sigma^{\prime}}(\mathbf{r}, \omega)
\end{aligned}
$$

where $\nu, \kappa$ are Cartesian indices. In Eq. (9),

$$
f_{\mathrm{XC}, \sigma \sigma^{\prime}}^{\mathrm{ALDA}}\left(\mathbf{r}, \mathbf{r}^{\prime}\right)=\left.\delta\left(\mathbf{r}-\mathbf{r}^{\prime}\right) \frac{d^{2} e_{\mathrm{XC}}^{h}\left(\bar{n}_{\uparrow}, \bar{n}_{\downarrow}\right)}{d \bar{n}_{\sigma} d \bar{n}_{\sigma^{\prime}}}\right|_{\bar{n}_{\uparrow, \downarrow}=n_{0 \uparrow, \downarrow}(\mathbf{r})}
$$

is the frequency-independent $\mathrm{XC}$ kernel associated with the adiabatic local-density approximation (ALDA), where $e_{\mathrm{xc}}^{h}$ is the xc energy density of a homogeneous electron gas and $n_{0 \sigma}$ the ground-state spin density of the system. The other terms in Eq. (9) represent nonadiabatic XC contributions, which bring in the dissipation. In the second term, $\sigma_{\nu \kappa, \sigma \sigma^{\prime}}^{\mathrm{XC}}$ is the spin-resolved viscoelastic stress tensor of the electron liquid. ${ }^{15}$ The key quantity in the last term of Eq. (9) is $\rho_{\uparrow \downarrow}$.

We now consider a specific excitation $p \sigma \rightarrow q \sigma$ between the Kohn-Sham levels $\psi_{p \sigma}$ and $\psi_{q \sigma}$, and assume the ground 
state to be spin unpolarized. To derive the TDDFT correction to the bare Kohn-Sham excitation energy $\hbar \omega_{p q \sigma}$, we apply the so-called small-matrix approximation. ${ }^{16,17}$ The result is, to lowest order in the nonadiabatic corrections,

$$
\begin{aligned}
\hbar^{2} \omega_{ \pm \sigma}^{2}= & \hbar^{2} \omega_{p q \sigma}^{2}+2 \hbar \omega_{p q \sigma}\left[\left(S_{\sigma \sigma}^{\mathrm{H}+\mathrm{ALDA}} \pm S_{\bar{\sigma} \sigma}^{\mathrm{H}+\mathrm{ALDA}}\right)\right. \\
& \left.+\left(S_{\sigma \sigma}^{\mathrm{VE}} \pm S_{\bar{\sigma} \sigma}^{\mathrm{VE}}\right)+\left(S_{\sigma \sigma}^{\mathrm{SCD}} \pm S_{\bar{\sigma} \sigma}^{\mathrm{SCD}}\right)\right],
\end{aligned}
$$

where the + or - sign refers to charge-or spin-density excitations (CDE or SDE), respectively. $S_{\sigma \sigma^{\prime}}^{\mathrm{H}+\mathrm{ALDA}}, S_{\sigma \sigma^{\prime}}^{\mathrm{VE}}$, and $S_{\sigma \sigma^{\prime}}^{\mathrm{SCD}}$ are the dynamical many-body corrections to the bare transition energy $\hbar \omega_{p q \sigma}$ between the single-particle levels $p \sigma$ and $q \sigma$. The Hartree + ALDA shift is given by

$$
\begin{aligned}
S_{\sigma \sigma^{\prime}}^{\mathrm{H}+\mathrm{ALDA}}= & \int d^{3} r \int d^{3} r^{\prime} \psi_{p \sigma}(\mathbf{r}) \psi_{q \sigma}(\mathbf{r}) \psi_{p \sigma^{\prime}}\left(\mathbf{r}^{\prime}\right) \psi_{q \sigma^{\prime}}\left(\mathbf{r}^{\prime}\right) \\
& \times\left(\frac{1}{\left|\mathbf{r}-\mathbf{r}^{\prime}\right|}+f_{\mathrm{XC}, \sigma \sigma^{\prime}}^{\mathrm{ALDA}}\left(\mathbf{r}, \mathbf{r}^{\prime}\right)\right),
\end{aligned}
$$

which causes no dissipation, $f_{\mathrm{xc}, \sigma \sigma^{\prime}}^{\mathrm{ALD}}$ being frequency independent and real. The viscoelastic (VE) shift is given by

$$
S_{\sigma \sigma^{\prime}}^{\mathrm{VE}}=\frac{i \omega}{\omega_{p q \sigma}^{2}} \sum_{\nu \kappa} \int d^{3} r \sigma_{\kappa \nu, \sigma \sigma^{\prime}}^{\mathrm{XC}, p q}(\mathbf{r}, \omega) \boldsymbol{\nabla}_{\kappa}\left(\frac{j_{p q \sigma, \nu}(\mathbf{r})}{n_{\sigma}(\mathbf{r})}\right),
$$

where $\sigma_{\kappa \nu, \sigma \sigma^{\prime}}^{\mathrm{XC}, p q}$ is the XC stress tensor ${ }^{14-16}$ with the exact current $\mathbf{j}_{\sigma, \nu}$ replaced by $\mathbf{j}_{p q \sigma}(\mathbf{r}) \equiv\left\langle\psi_{p \sigma}\left|\hat{\mathbf{j}}_{\sigma}\right| \psi_{q \sigma}\right\rangle$, with $\hat{\mathbf{j}}_{\sigma}$ the paramagnetic particle current density operator. Equation (13) can be viewed as the average rate of energy dissipation per unit time in a viscous fluid, where $\sigma_{\kappa \nu, \sigma \sigma^{\prime}}^{\mathrm{XC}, p q}$ is the viscoelastic stress tensor of the fluid, and $\boldsymbol{\nabla}_{\kappa}\left[j_{p q \sigma, \nu} / n_{\sigma}\right]$ the velocity gradient. In contrast to the familiar expression from classical fluid dynamics, ${ }^{18} S^{\mathrm{VE}}$ has both real and imaginary parts.

The SCD shift is a central result of this paper:

$$
\begin{aligned}
S_{\sigma \sigma}^{\mathrm{SCD}} \pm S_{\bar{\sigma} \sigma}^{\mathrm{SCD}}= & \frac{i e^{2} \omega}{\omega_{p q \sigma}^{2}} \int d^{3} r \rho_{\uparrow \downarrow}\left(\omega ; n_{\uparrow}(\mathbf{r}), n_{\downarrow}(\mathbf{r})\right) \\
& \times\left(\frac{n_{\bar{\sigma}}(\mathbf{r})}{n_{\sigma}(\mathbf{r})}\left|\mathbf{j}_{p q \sigma}(\mathbf{r})\right|^{2} \mp \mathbf{j}_{p q \bar{\sigma}}(\mathbf{r}) \cdot \mathbf{j}_{p q \sigma}(\mathbf{r})\right) .
\end{aligned}
$$

As we will show in an example below, under certain circumstances this new contribution to the broadening of an excitation can actually dominate the damping process.

By comparison with Eqs. (4) and (5), we immediately recognize the structure of the power loss typical of the Coulomb drag force. ${ }^{19}$ Like the viscoelastic term (13), the SCD term (14) contains both a real and an imaginary part. Notice that if the external driving force couples in a different way to the two spin components, such that the average spin velocities are different, the SCD term contributes to the charge channel too. In this particular case the two spin populations may be considered distinguishable, characterized by a spindependent frequency $\omega_{\sigma}$ in both the charge and the spin channel. This implies that the Coulomb drag force exerted by one population onto the other can be regarded as an external force.

This concept can be clarified by considering the intersubband charge and spin plasmons in a quantum well. ${ }^{20-22}$ The

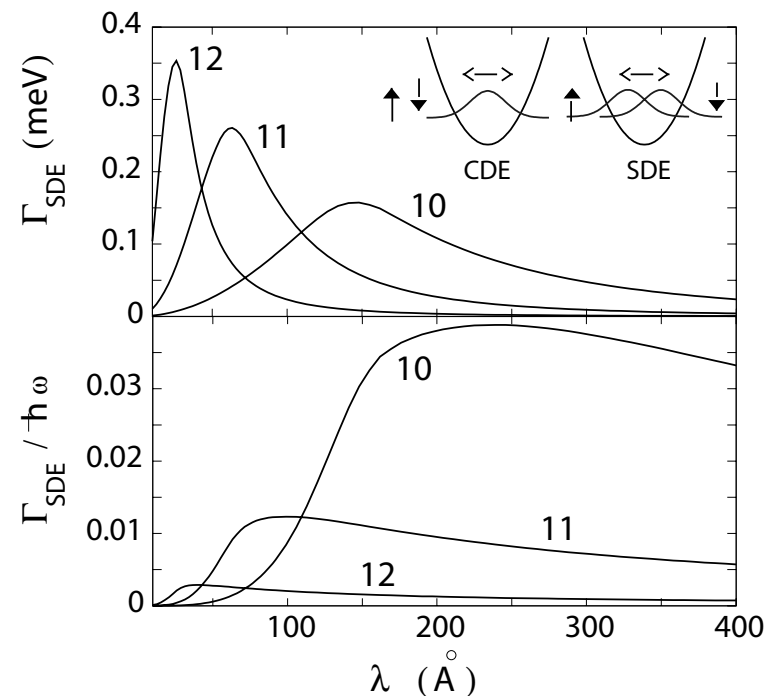

FIG. 2. Upper panel: Spin-plasmon linewidth $\Gamma_{\mathrm{SDE}}^{\mathrm{SCD}}$ for a parabolic quantum well versus curvature parameter $\lambda$, for $N_{s}=10^{10}$, $10^{11}$, and $10^{12} \mathrm{~cm}^{-2}$ and GaAs parameters. The inset illustrates the collective motion of the two spin populations (CDE, in phase; SDE, out of phase). Lower panel: Rescaled linewidth $\Gamma_{\mathrm{SDE}}^{\mathrm{SCD}} / \hbar \omega$.

inset to Fig. 2 illustrates the two types of density oscillation for a parabolic well, in which the $n_{\uparrow}$ and $n_{\downarrow}$ components move back and forth, perpendicular to the $x y$ plane of the quantum well, in phase (CDE) or with opposite phase (SDE). In the case of the SDE, the average net momentum transferred by Coulomb interactions from the $\bar{\sigma}$ - to the $\sigma$-spin population is directed opposite to the $\sigma$-spin direction of motion, so that the SCD effect damps the motion of both spin populations. For the charge plasmon the effect can become more subtle: since the average spin velocities are in the same direction, the net result of Coulomb interactions between the two spin populations will be to transfer momentum from the "hotter" to the "colder" population, until equilibrium is reached. In this case the SCD effect will not damp the motion of both spin populations, but pump momentum from the faster to the slower.

We now proceed to estimate the size of the SCD effect for optical excitations in a parabolic quantum well. According to the harmonic potential theorem, ${ }^{23}$ the intrinsic linewidth of a $\mathrm{CDE}$ in a parabolic confining potential is strictly zero. The TDDFT linear response equation (7) satisfies this requirement: CDE's in a parabolic well have a uniform velocity profile, so that the viscoelastic stress tensor vanishes. Likewise, in expression (13) for $S_{\sigma \sigma^{\prime}}^{\mathrm{VE}}, \nabla_{\kappa}\left[j_{p q \sigma, v} / n_{\sigma}\right]$ is very small. The viscoelastic contributions to SDE's are thus a higherorder correction compared to the SCD contributions, which give the dominant correction to the excitation frequency beyond the ALDA. The intrinsic SDE linewidth for a parabolic quantum well therefore becomes $\Gamma_{\mathrm{SDE}} \approx \Gamma_{\mathrm{SDE}}^{\mathrm{SCD}}$, where

$$
\begin{aligned}
\Gamma_{\mathrm{SDE}}^{\mathrm{SCD}}(\omega)= & \frac{e^{2} N_{s} \omega}{2 \omega_{p q \sigma}^{2}} \int d z \operatorname{Re} \rho_{\uparrow \downarrow}\left(\omega ; n_{\uparrow}(z), n_{\downarrow}(z)\right) \\
& \times\left(\frac{n_{\bar{\sigma}}(z)}{n_{\sigma}(z)}\left|\mathbf{j}_{p q \sigma}(z)\right|^{2}+\mathbf{j}_{p q \bar{\sigma}}(z) \cdot \mathbf{j}_{p q \sigma}(z)\right),
\end{aligned}
$$

with $N_{s}$ the two-dimensional electronic sheet density. 
Numerical results for $\Gamma_{\mathrm{SDE}}^{\mathrm{SCD}}$ for a GaAs-based quantum well are shown in Fig. 2. We assume only the first subband to be occupied, i.e., $n_{\sigma}(z)=N_{s}\left|\psi_{1 \sigma}(z)\right|^{2}$, and approximate the Kohn-Sham orbitals $\psi_{q, p \sigma}(z)$ entering Eq. (15) by the first two eigenstates of a harmonic oscillator with external potential $\hbar^{2} z^{2} / 2 m \lambda^{4}$. Furthermore, to lowest order in the nonadiabatic corrections $\omega_{\sigma}$ can be replaced with $\omega_{p q \sigma}$. For this system the parameters that govern the linewidth of the SDE mode are $N_{s}$ and the quantum well curvature parameter $\lambda$. The latter determines both the excitation frequency and the characteristic width of the ground-state density distribution. The results in Fig. 2 show that $\Gamma_{\mathrm{SDE}}^{\mathrm{SCD}}$ can be nonnegligible (a large fraction of $\mathrm{meV}$ ) for experimentally reasonable parameters, ${ }^{24}$ and $\Gamma_{\mathrm{SDE}}^{\mathrm{SCD}} / \hbar \omega$ can be of the order of a few percent for a large range of curvature parameters and carrier densities.

For a specific $N_{s}$, the linewidth exhibits a well-defined maximum as a function of $\lambda$. The position of this maximum is determined by the competition of two distinct effects: (i) The low-density saturation value of $\rho_{\uparrow \downarrow}$ increases with $\lambda$ [i.e. decreases with $\omega$; see Eq. (6)]; (ii) the average particle velocity decreases with $\lambda$ (i.e., decreases with the parabolic curvature). The two effects give opposite contributions to the dissipation [see Eq. (3)], and the maximum occurs when the second effect takes over. Due to the density dependence of $\rho_{\uparrow \downarrow}$ (see Fig. 1), a substantial contribution to the integrand in Eq. (15) can come from the lateral regions of the quantum well, where the particle density is low. This is in contrast to the VE contribution, which tends to be dominated by the high-density regions.

The above example shows that, even when other forms of damping, such as disorder and phonons, are drastically reduced by careful selection of the system characteristics, the dissipation induced by SCD cannot be avoided, due to its intrinsic nature.
Equation (15) suggests an experimental way to extract the impact of SCD on spin dynamics, namely, by an optical measurement of the linewidth of both charge and spin plasmons in the same parabolic quantum well. Such an experiment can be carried out using inelastic light scattering. ${ }^{25}$ Under the reasonable assumption that (i) extrinsic (ext) damping (nonmagnetic impurities, phonons) affect the CDE and SDE in the same way, and (ii) the viscoelastic term can be disregarded due to the parabolic system geometry, we have

$$
\Gamma_{\mathrm{SDE}}-\Gamma_{\mathrm{CDE}} \approx\left(\Gamma_{\mathrm{SDE}}^{\mathrm{ext}}+\Gamma_{\mathrm{SDE}}^{\mathrm{SCD}}\right)-\left(\Gamma_{\mathrm{CDE}}^{\mathrm{ext}}\right) \approx \Gamma_{\mathrm{SDE}}^{\mathrm{SCD}},
$$

i.e., the SCD contribution to the spin-plasmon linewidth is given to a very good approximation by the difference between the SDE and the CDE linewidths. This provides a valuable opportunity for comparison with microscopic models for the transresistivity via Eq. (15), using the appropriate Kohn-Sham single-particle orbitals of the system.

In conclusion, we have presented a discussion of the power loss in a device due to dissipation of spin-dependent currents induced by SCD forces. We have suggested a purely optical method to measure the SCD effect in spin-density excitations in parabolic quantum wells. In the $\omega \rightarrow 0$ limit, a particularly interesting application of our formalism would be to describe the SCD intrinsic dissipation in spindependent transport through single-molecular junctions. ${ }^{26} \mathrm{As}$ the broad effort in spintronics, quantum computation, and transport in micro- and mesoscopic systems continues, we expect a growing impact of the SCD effect in future applications.

This work was supported by DOE Grant No. DE-FG0205ER46213, NSF Grant No. DMR-0553485, Research Corporation, the Nuffield Foundation Grant No. NAL/01070/G, and by the Research Fund 10024601 of the Department of Physics of the University of York.
*Electronic address: ida500@york.ac.uk

${ }^{1}$ I. D'Amico and G. Vignale, Phys. Rev. B 62, 4853 (2000).

${ }^{2}$ I. D'Amico and G. Vignale, Europhys. Lett. 55, 566 (2001).

${ }^{3}$ I. D'Amico and G. Vignale, Phys. Rev. B 65, 085109 (2002).

${ }^{4}$ K. Flensberg, T. S. Jensen, and N. A. Mortensen, Phys. Rev. B 64, 245308 (2001).

${ }^{5}$ I. D'Amico and G. Vignale, Phys. Rev. B 68, 045307 (2003).

${ }^{6}$ C. P. Weber et al., Nature (London) 437, 1330 (2005).

${ }^{7}$ J. M. Kikkawa and D. D. Awschalom, Phys. Rev. Lett. 80, 4313 (1998); J. M. Kikkawa and D. D. Awschalom, Nature (London) 397, 139 (1999).

${ }^{8}$ G. Schmidt et al., Phys. Rev. B 62, R4790 (2000).

${ }^{9}$ This is particularly important when spin-dependent applications in quantum information and computation are considered: operation (gating) times must in fact be at least $10^{-4}$ times smaller than the spin-decoherence times. See, e.g., M. A. Nielsen and I. L. Chuang, Quantum Computation and Quantum Information (Cambridge University Press, Cambridge, 2003), pp. 480-482.

${ }^{10}$ J. M. Kikkawa and D. D. Awschalom, Science 287, 473 (2000); J. A. Gupta et al., ibid. 292, 2458 (2001).

${ }^{11}$ M. Petersilka et al., Phys. Rev. Lett. 76, 1212 (1996).
${ }^{12}$ G. Vignale and W. Kohn, Phys. Rev. Lett. 77, 2037 (1996).

${ }^{13}$ R. D'Agosta and G. Vignale, Phys. Rev. Lett. 96, 016405 (2006).

${ }^{14}$ G. Vignale et al., Phys. Rev. Lett. 79, 4878 (1997).

${ }^{15}$ Z. Qian et al., Phys. Rev. Lett. 90, 066402 (2003).

${ }^{16}$ C. A. Ullrich and K. Burke, J. Chem. Phys. 121, 28 (2004).

${ }^{17}$ H. Appel et al., Phys. Rev. Lett. 90, 043005 (2003).

${ }^{18}$ L. D. Landau and E. Lifshitz, Mechanics of Fluids, Course of Theoretical Physics Vol. 6 (Pergamon Press, Oxford, 1987).

${ }^{19}$ As for the sign, we emphasize that the Kohn-Sham single-particle currents $\mathbf{j}_{p q \sigma}$ should not be confused with the velocities of the interacting system. In particular, the former can have a different direction with respect to the the actual system current.

${ }^{20}$ I. K. Marmorkos and S. Das Sarma, Phys. Rev. B 48, 1544 (1993).

${ }^{21}$ C. A. Ullrich and M. E. Flatté, Phys. Rev. B 66, 205305 (2002).

${ }^{22}$ C. A. Ullrich and M. E. Flatté, Phys. Rev. B 68, 235310 (2003).

${ }^{23}$ J. F. Dobson, Phys. Rev. Lett. 73, 2244 (1994).

${ }^{24}$ These results for $\Gamma_{\mathrm{SDE}}^{\mathrm{SCD}}$ correspond to picosecond lifetimes, which are indeed very short compared to typical spin-flip times.

${ }^{25}$ A. Pinczuk et al., Phys. Rev. Lett. 63, 1633 (1989).

${ }^{26}$ N. Sai et al., Phys. Rev. Lett. 94, 186810 (2005). 